Document downloaded from:

http://hdl.handle.net/10251/102349

This paper must be cited as:

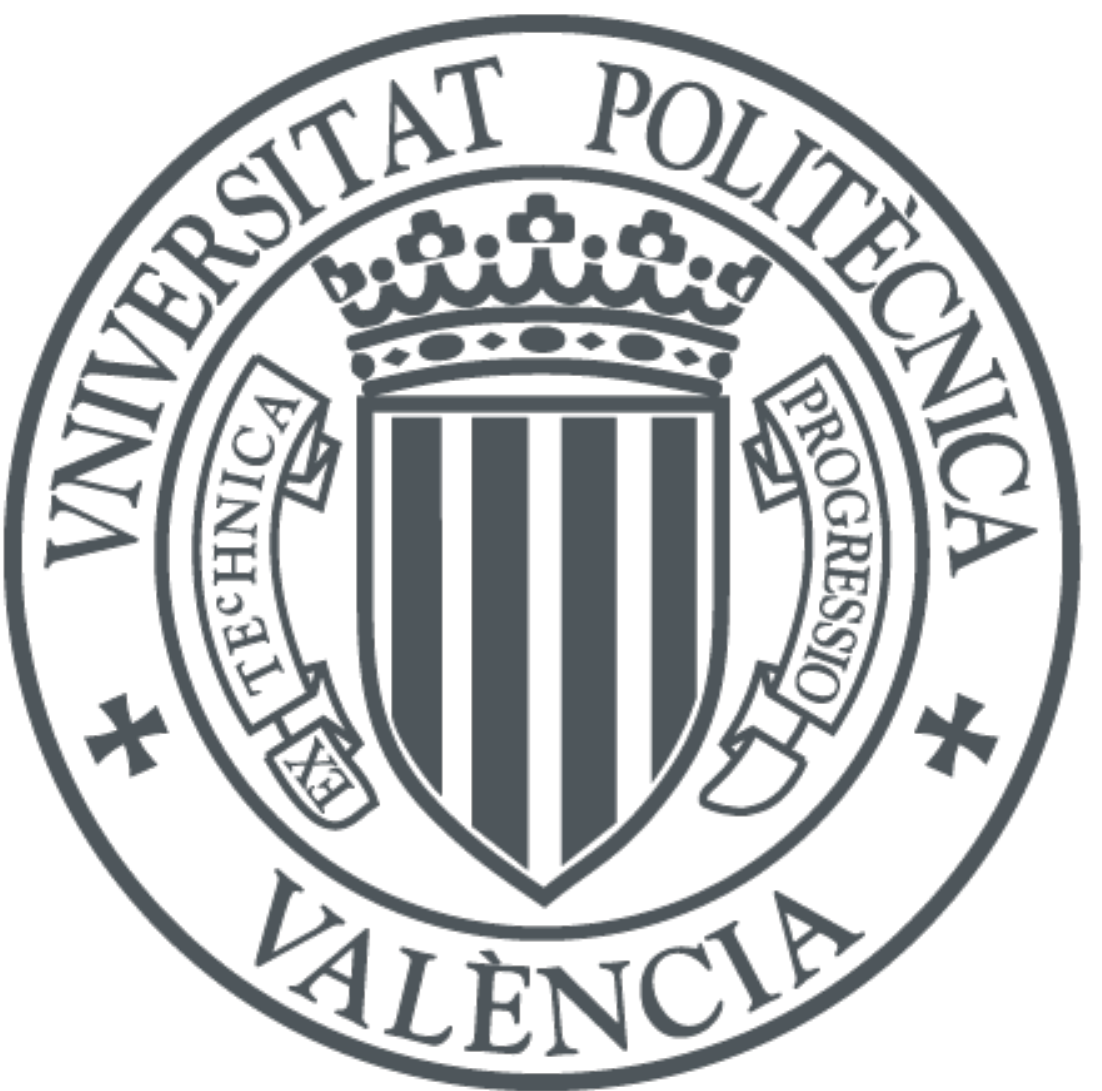

The final publication is available at

https://doi.org/10.1007/s11227-017-2214-4

Copyright Springer-Verlag

Additional Information 


\title{
System for monitoring the wellness state of people in domestic environments employing emoticon-based HCI
}

\author{
Laura García, Lorena Parra, Oscar Romero, Jaime Lloret \\ Instituto de Investigación para la Gestión Integrada de zonas Costeras \\ Universitat Politècnica de València. C/ Paranimf, 146730 Grau de Gandia, Valencia, Spain \\ laugarg2@teleco.upv.es, loparbo@doctor.upv.es, oromero@dcom.upv.es, jlloret@dcom.upv.es
}

\begin{abstract}
Wellness state is affected by the habitability state of the domestic environment. Monitoring it can help to discover the causes of a low wellness levels aiding people in the improvement of their quality of life. In this paper, we propose a system to monitor the wellness state of people utilizing Liketrt's scale to determine the state of the user through an emoticon-based Human-Computer Interaction (HCI). The system is intended for domestic environments and measures the habitability conditions of the dwelling (such as temperature, humidity, luminosity and noise) employing sensors. An algorithm is designed in order to establish how to measure those conditions and to calculate the statistics that allows tracking their progress. The obtained information is presented to the user to compare his/her wellness state with the habitability conditions. Measures in a real domestic environment were performed in order to determine the configuration of our system. The energy-efficiency of the algorithm provides an improvement between $99.36 \%$ and $99.62 \%$ in the energy consumption depending on the selected parameters.
\end{abstract}

Keywords - Human Computer Interaction; wellness state; habitability; e-health; indoor monitoring

Acknowledgements

This work has been partially supported by the "Ministerio de Ciencia e Innovación", through the "Plan Nacional de I+D+i 2008-2011" and by the "Ministerio de Educación, Cultura y Deporte", through the grand "Ayudas para contratos predoctorales de Formación del Profesorado Universitario FPU14/02953".

\section{INTRODUCTION}

The awareness on the wellness state of people has been gradually increasing throughout the years. There are many e-health monitoring proposals that allow to determine the evolution of the state of a patient [12]. But as life expectancy increases, the next concern is how to live a rewarding life and improve the wellbeing of people [3]. In 1950, Halbert L. Dunn introduced the concept of wellness as we use it nowadays [4]. Both physical and mental states are some of the aspects that greatly affect the wellness state of a person. However, these aspects can, in turn, be compromised by external agents.

The domestic environment can significantly influence the health state of the inhabitants of the dwelling and their wellness state [5]. The Organisation for Economic Co-operation and Development (OECD) includes housing conditions on its survey about wellbeing [6]. Some of the habitability factors they address on what affects the wellness state of the people are dampness, exposure to noise, and indoor air quality. Indoor temperature is another factor that can cause a decrease in the health of the people. Many researches study the effects of temperature on respiratory diseases [7-8] but temperature is also the cause of other afflictions. Because the heat waves are more and more frequent, each year, the number of people affected by them increases. In countries that usually experience high temperatures, news on people affected by heat strokes or its symptoms are constantly on the media. In 2003, the number of deaths by heat stroke in Spain reached 169 [9]; however, the number of people With any condition related to their 
well-being due to these heat waves is much higher. Not having good housing conditions increase the probability of developing bad physical and psychological conditions leading to an increase in health costs such as hospitalizations and prescriptions, which is paid by the government or civilians themselves depending on the country [10]. The aforementioned reasons manifest the importance of measuring the wellbeing of people in domestic environments. Still, in order to determine the wellness state of the inhabitants of a house the interaction between the system and the user is needed.

Human-Computer Interaction (HCI) between users and e-health applications should be fluid and easy. These applications can be employed by people with health states ranging from an outstanding health to patients with chronic illnesses, severe pain or with reduced mobility, among other conditions. In order to provide the best Quality of Experience (QoE) when utilizing an application, factors such as the difficulty of employing the application, its performance, the appearance or the time it takes to learn how to use the system have to be considered [11]. Nowadays, emoticons are employed every day to provide emotions to express feelings and compose communications where it is not possible to interact with a human. The emotions the emoticons portray are widely known and its use has gained so much popularity. Because of that, the available icons have been increasing through the years and social media is offering a wider selection. Including emoticons in HCI allows to obtain a more accurate description of the state of the user as well as to portray information in a more meaningful way [12].

In this paper, a system that monitors the quality of life of the people in domestic environments is proposed. It presents a sensor network that measures habitability conditions of a domestic environment and provides the information to the user. The system also presents an HCI functionality that allows determining the wellness state of the user by selecting the emoticon that represents a certain state. These emoticons are accompanied with the name of the state in order to better identify a certain condition. This allows having a two-way communication where the user provides his or her condition to the system and the system provides the information on the habitability conditions as well as the development of the wellness state of the user. We also perform measures in a real setting and determine the energy consumption of the data forwarding of our system. Our contribution highlights are:

- Proposal of a monitoring system to measure the quality of life of the user.

- Development of an algorithm to improve the energy efficiency of the system.

- The prototype of the habitability monitoring sensor network that communicates with the system.

- An HCI functionality that interacts with the users both for knowing their wellness state and to inform them of their wellness state and the habitability conditions of their home.

This system can be deployed in different indoor environments. Not only in dwellings but also in hospitals, nursing homes, hotels, cruises or any other enclosed environment where it is important to know and improve the wellness state of the people.

The rest of the paper is organized as follows. The related work is presented in Section 2. Section 3 presents the proposal of our system. The HCI functionality is depicted in section 4. Results are discussed in section 5. Lastly, our conclusion and future work are provided in Section 6.

\section{RELATED WORK}

In this section, the related work is discussed. The main aspects considered in this section are e-health and wellness monitoring technologies, the effects of the state of the dwelling and its consequences on the wellbeing of its inhabitants and HCI applied to e-health applications.

Technologies focusing on e-health and specifically on wellness are getting more and more popular. Nowadays many studies are introducing it to their proposals. A priority-based protocol for assisted living is proposed by Hemant Ghayvat et al. in [13]. It performs real-time analysis, data streaming, decisionmaking and control functionalities. They obtain a reduction ranging from $60 \%$ to $72 \%$ of the data in comparison with ZigBee. Abdur Rahim Mohamed Forkan et al. present in [14] a wellness monitoring system for adults suffering from cardiac diseases. Social media is employed to provide the health condition of the patient to his or her contacts. This information is captured by electrocardiogram (ECG) sensors and a fitbit device. In the event of abnormal readings, a notification is sent through Facebook to the contacts of the patient. An application to improve the mental health of students is implemented in [15] 
by Chad Errol R. Booc et al. It provides anonymous mental aid to its users. The application also gathers information of the demographics of the users in order to obtain statistics on the overall mental state of the campus. Wajahat Ali Khan et al. propose in [16] PULSE (Personalized Ubiquitous Life-care Decision Support System). It employs wellness information gathered from clinical data and life style information to provide a personalized recommendation. A system that provides recommendations of suitable physical activity to improve wellbeing is presented in [17] by Chae-Gyun Lim et al. In order to do so, they employ information on the daily activities of the user utilizing wearable sensors and a smartphone. They also apply an algorithm in order to suggest the best physical activity. Bengisu Tulu et al. propose in [18] an application to improve the wellness state of adults suffering from type 2 diabetes. An application that predicts cardiac problems by gathering heart rate information through a wearable device is presented by Damanpreet Kaur et al. in [19]. The application provides a recommendation of the necessary measures to take in order to improve the wellness state of the user. For that purpose, the recommendation provides advice in diet and physical activity. Atika Arshad et al. propose in [20] a monitoring system to measure vital signals and sleep patterns of elderly people. It is intended to be deployed inside the bedroom of the patient by placing movement sensors on the bed and temperature and humidity sensors by the door of the bedroom. The system also presents an alarm functionality in order to alert of an emergency situation.

Domestic habitability parameters are related to the wellness state of the inhabitants of the dwelling. Cladia J. Martin et al. perform in [21] a study of the effects of damp in physical and mental health. Results show that inhabitants of damp houses are prone to be more nervous and respiratory symptoms increase in children in comparison to those living in non-damp houses. The existence of mold worsens the condition of the people. A survey on mental health depending on the type of dwelling is presented by Gary W. Evans in [22]. They conclude that people living in multi-dwelling housing are more likely to suffer from bad psychological health, specifically anxiety. A review of the effects of housing in public health is presented in [23]. Mary Shaw suggests that cold temperatures, dampness and lack of ventilation are related to respiratory diseases. She also relates extreme cold temperatures to a raise of blood pressure, serum cholesterol and myocardial infarctions in extreme cases. There is also a higher number of elderly deaths during winter. Indoor air pollution, such as radon, is related to lung dysfunctions and lung cancer. Hilary Thomson et al. perform in [24] a survey that relates housing and health. Results show that better temperature conditions inside the house lead to a better relationship between inhabitants. It also improves respiratory health problems, general health problems and mental health. They state that the impact of better conditions is very significant to those with chronic respiratory diseases. A study on skin conditions of farm workers due to housing conditions is presented by Cheryl J. Gustafson et al. in [25]. They conclude that puritus cases were more likely to happen to people living without air conditioning, rash was related to low humidity and scaling happened with dwelling temperatures in the thermal discomfort range. Raquel Laquesta et al. implemented in [26] a system that recommends the best neighborhood to live in based on the wellness state of the user. For that purpose, they employ a wearable device to measure Heart Rate Variability (HRV) while visiting several housing options. Then, the data is analyzed by an algorithm that provides the recommendation of the dwelling that allows the users to have the best wellness state possible.

Human Computer Interactions (HCI) in e-health applications are of great importance due to the impact they can have on the wellness state of the patient. There have been several proposals on HCI systems for both e-health and other applications. Fatima Isiaka et al. propose in [27] a HCI model that employs nonobstructive techniques in order to predict the behavior of the user. They present an algorithm to measure the interaction and stress levels of the user employing the data collected from Skin Conductance Measures (SCM). Results show that the performance of the model is accurate enough to distinguish the patterns of the users. An HCI system that employs eye movement and Electroencephalogram (EEG) signals to provide Locked-In Syndrome (LIS) patients with a form of communication is proposed by Shining Han et al. in [28]. The system allows patients to use their eye movement to perform the functions of a mouse or a touchpad. The EEG signals provide the information on whether the user is looking at the display consciously or not. The results show an average accuracy of $85 \%$. Bo-Hao Chan et al. propose in [29] an HCI system for cars in order to prevent driver distraction. They have considered an intuitive interaction framework and a transparent windshield display and they address the difficulties of both solutions. Participants gave an average Mean Opinion Score (MOS) of 3.71 for Ease of Use, 3.62 for Usefulness and 3.57 for Non-distracting. A technique for feature extraction employing 2D-LDA and 2DPCA is presented by Shaaib Kamal et al. in [30]. They employed the Support Vector Machine (SVM) and K-Nearest Neighborhood (KNN) classifiers and obtained a $97.63 \%$ accuracy for the JAFFE database and 
94.8\% accuracy for the Cohn-Kanade database. Lastly, Rishabh Agrawal et al. propose in [31] a method to recognize hand movements and gestures in order to interact with computers. It uses a Senz3D camera and analyzes the 3D data to classify hand gestures. It is able to replace devices such as keyboards and mice. They conclude that the propose method outperforms existing ones.

Our proposal compiles e-health monitoring, domestic environment monitoring and HCI aspects presenting a wellness monitoring system based on domestic habitability parameters that includes a dynamic and easy to use HCI. The HCI functionality allows providing a two-way feedback between the user and the system. With our contribution, we provide the means to study in detail the effects of the conditions of the dwelling on the wellness state of its inhabitants providing a new technology to improve the overall quality of life of people.

\section{PROPOSAL}

In this section, the habitability parameters we have selected are detailed as well as its effects on health. The topology of the system will be depicted as well. An algorithm to establish the method of acquiring measures are proposed as well. Lastly, we present the mathematical model for calculating the energy expense of our system.

\subsection{Habitability parameters}

The habitability parameters employed in this system are described in this section. The way people can be affected both psychologically and physically by those parameters is presented as well.

There is a large quantity of researches on the effects of different habitability parameters and its effects on the health of the inhabitants of the dwelling. We have selected the parameters that we considered to be the most significant.

- Temperature: Temperature changes can have a great impact on health. Recently, Europe is facing more and more heat waves and cold fronts leading to unusual temperature conditions that many households are not able to face economically [32]. It results in a decrease of the wellness state of the inhabitants of the house as well as a decrease of their health. Extreme temperatures lead to an inadequate response of the thermoregulatory system, a high loss of water and electrolytes needed for the correct operation of the organs, cramps, dehydration, heat strokes, motion instability, convulsions and coma [33]. Children and elderly people as well as people with chronic illnesses, cardiovascular, respiratory and mental illnesses, some medical treatments or people under the effects of alcohol and drugs are at risk with extreme temperatures.

- Humidity: Dampness in domestic environments is related to cough, wheeze and asthma as well as tiredness, headache and upper airway infections [34]. It also can lead to the apparition of mold and fungal spores, which can produce allergies, asthma and other respiratory symptoms such as waking because of shortness of breath or chest tightness [35].

- Luminosity: Having inadequate luminosity leads to changes in the circadian rhythm affecting the quality of sleep [36]. High luminosity increases the tendency of doing physical activity, whether low exposure to daylight decreases vitality [37]. Good lighting also improves the condition in people with stress and the overall mood of a person [38], as well as some symptoms of Alzheimer's disease [39] and depression [40].

- Noise: Noise can affect people on various levels depending on the moment of the day. Usually, at nighttime people are more sensitive to noise than at daytime. Because of that, many of the effects caused by noise are related to sleep deprivation [41]. It is also associated with higher stress which can develop into an increase in the cardiovascular risk.

\subsection{Topology}

In this subsection, the topology of the system is presented. The message exchange between the elements of our system is also depicted considering the different cases that can occur depending on the variation of the habitability parameters.

The topology of our system is presented in Fig. 1. The sensor node is located in a spacious place without 
being covered by any furniture. The habitability parameters measured by the sensors are delivered to the database. It stores both the habitability parameters and the data on the wellness state of the user. The smartphone receives the information from the database and obtains the wellness state of the user with the HCI based on emoticons. This allows having a two-way interaction between the user and the system that permits the correlation between the measured metrics. Doing so, users can visualize the progress of their wellness state as well as the statistics on the habitability parameters on their smartphones.

The messages exchanged between the devices and the database are presented in Fig. 2. Firstly, the threshold and forwarding time settings are transmitted to the sensor node. Every time a message is received an acknowledgement message (ACK) is sent in order to confirm the information has reached its destination. The sensor node measures the habitability parameters, calculates the statistics and sends the results to the database. The database sends the results in order to be visualized on the smartphone. The smartphone is asked for the wellness state and after the user has indicated his or her state, the data is forwarded to the database. The wellness statistics calculations are done and the results are forwarded to the smartphone in order for them to be accessed by the user. When the parameters have not changed, the sensor node sends a message specifying to maintain the values. Then, the statistics are updated.

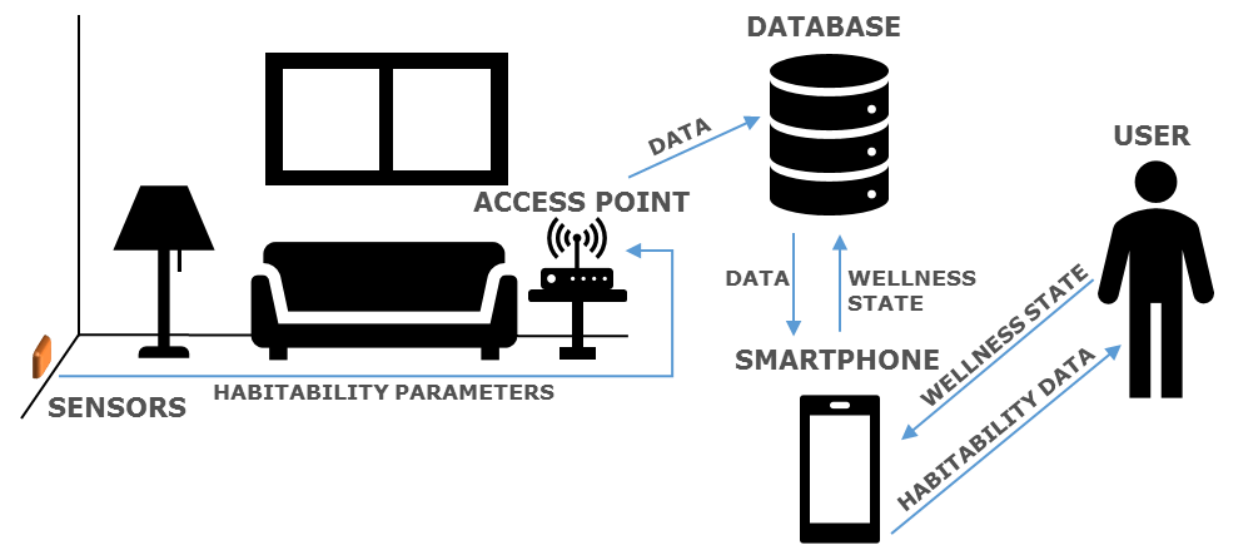

Fig. 1. Topology of the system. 


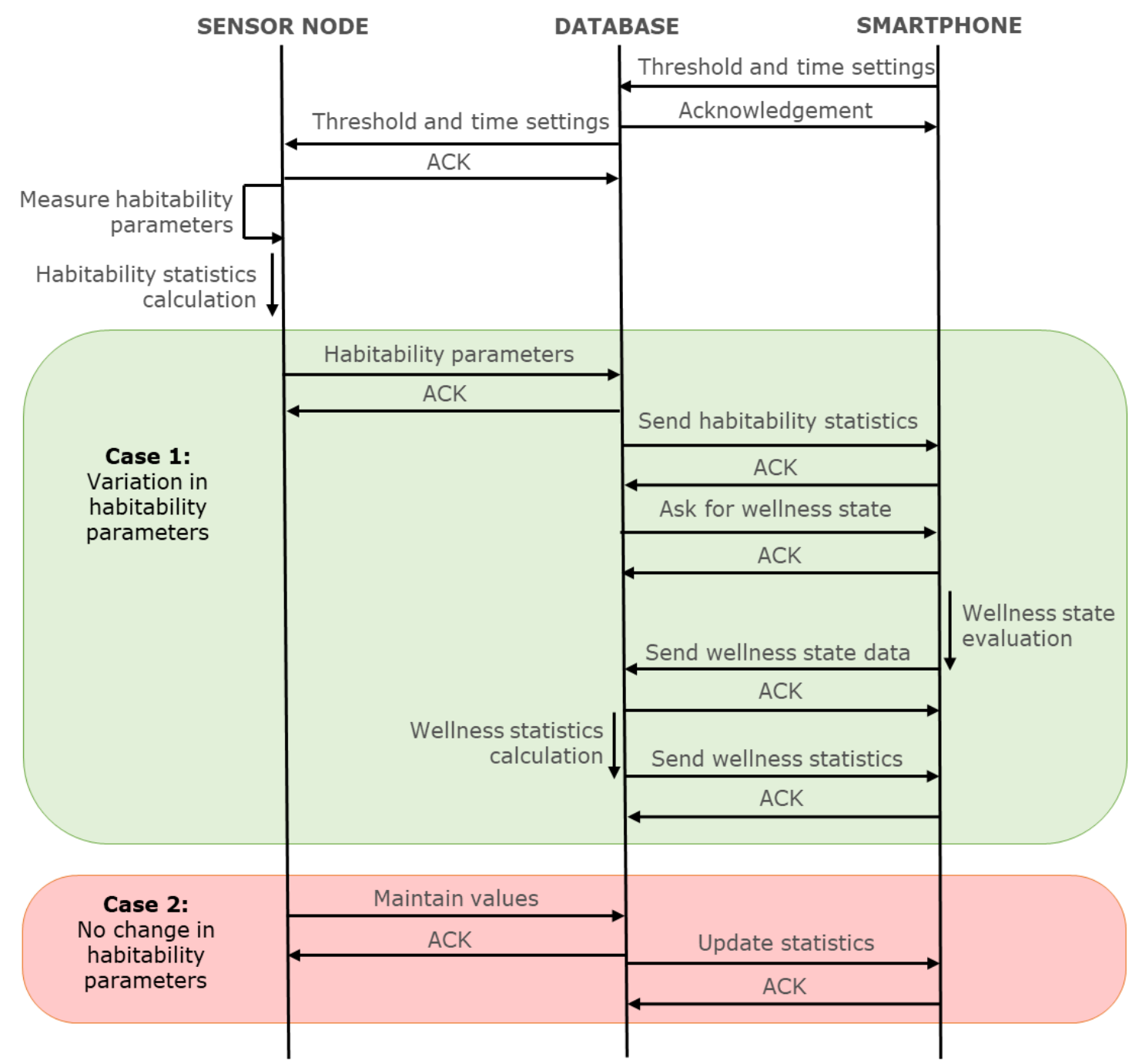

Fig. 2. Message exchange between the elements of the system.

\subsection{Habitability measures}

The process of acquiring the data on the habitability parameters is described in this subsection. An algorithm that measures the habitability parameters and decides when to send the information is presented. The configuration of the data in the IPv6 packet is also presented.

In order to measure the habitability parameters, we have employed temperature, humidity, luminosity and noise sensors. The sensitivity of the sensors is $0.1^{\circ} \mathrm{C}$ for the temperature sensor, $0.1 \%$ for the humidity sensor, $1 \mathrm{lux}$ for the luminosity sensor and $1 \mathrm{~dB}$ for the noise sensor. As constantly forwarding the gathered data would consume a lot of resources, we have designed an algorithm to collect and decide the data that can be transmitted. Fig. 3 presents the proposed algorithm. Firstly, the system sets the thresholds for all the parameters. Although the default values are $5 \%$ for humidity, $0.5^{\circ} \mathrm{C}$ for temperature, 10 lux for luminosity and $5 \mathrm{~dB}$ for noise, the user could personalize the 


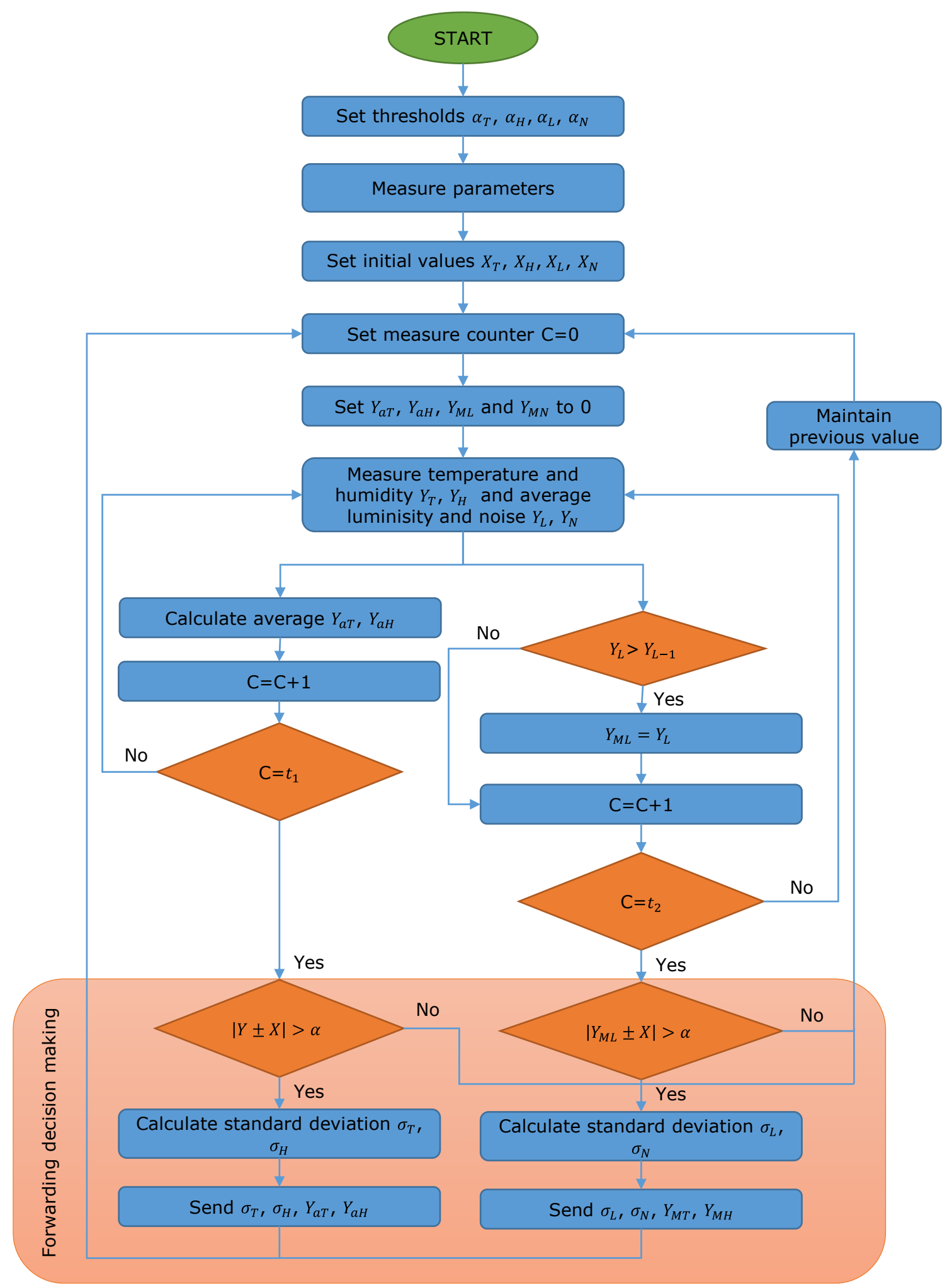

Fig. 3. Algorithm for measuring and forwarding decision making.

thresholds introducing in the system the desired values. Then, an initial measure is performed in order to establish the values for $X_{T}, X_{H}, X_{L}, X_{N} . X_{T}$ is the reference temperature value, $X_{H}$ is the reference humidity value, $X_{L}$ is the reference luminosity value and $X_{W}$ is the reference noise value. The measure counter is then initialized to cero and the maximum luminosity value, $Y_{M L}$, and maximum noise value, $Y_{M N}$, is also set to cero. For temperature and humidity, measures are performed each minute and forwarded at the time specified by the system thus, the measure count is provided by the database in order 
to transmit the data. For luminosity and noise, the measures are taken every 0.1 seconds. The average temperature, $Y_{\triangle T}$, average humidity, $Y_{\triangle H}$, maximum luminosity value, $Y_{M L}$, and maximum noise value, $Y_{M N}$, are set to cero as well. The measures are performed after that. Depending on the measure, the process is different. That is because for temperature and luminosity the average is calculated and for luminosity and noise, the maximum value is determined. The measure counter increases by one each time a measure is performed. When the counter reaches $t$, the algorithm reaches the forwarding decision making. $t_{1}$ and $t_{2}$ are different and the tests performed to determine their values are presented in Section 5.1. The obtained measures are compared with the reference value in order to determine if the threshold has been surpassed. If so, the standard deviation is calculated for both temperature and humidity and it is forwarded along the average temperature and humidity. If the threshold has not been reached, the previous value is maintained and the counter is set to cero again.

Now, we have to decide the codification of the information in the data portion of the IPv6 packet. The first parameter transmitted will be the noise. Noise can have values between 0 and $90 \mathrm{~dB}$, thus it will consume 7 bits to codify the data. The second parameter is the luminosity; the luminosity can present values between 0 and up to 100001 ux. To transmit it, it will be necessary to use 14 bits. The humidity is the third transmitted parameter, with values between 0 and $100 \%$. Therefore, 7 bits must be employed for humidity. The last variable will be the temperature; it can take values between -18 and $46^{\circ} \mathrm{C}$. According to the number of needed bits we will use one octet per parameter to transmit noise, humidity and temperature. For luminosity data two octets must be used, see Fig. 4.

\begin{tabular}{|l|c|c|c|c|}
\hline \multirow{2}{*}{ Octet } & 1 Byte & 2 Byte & 3 Byte & 4 byte \\
\hline \multirow{2}{*}{ Data } & Noise & \multicolumn{2}{|c|}{ Luminosity } & Humidity \\
\cline { 2 - 4 } & Temperature & \multicolumn{2}{|c}{} \\
\cline { 2 - 4 }
\end{tabular}

Fig. 4. Configuration of the data portion of the proposed system

\subsection{Analytical model}

In this subsection, the analytical model used to describe the energy consumption of our system is shown.

Based on [42], equation 1 to 5 can be used to estimate the energy consumed per bit in the transmitterreceiver model. It is assumed that energy dissipated in electronic transmission and reception is $50 \mathrm{~nJ} / \mathrm{bit}$, and $100 \mathrm{pJ} / \mathrm{bit} / \mathrm{m}^{2}$ in output transmission [43]. The maximum energy consumed in coding and decoding will be $0.55 \mathrm{~nJ} / \mathrm{bit}$, which corresponds to AES encryption [44].

$$
E_{\text {bit }}=E_{\text {bit }-t x}+E_{\text {bit -out }}+E_{\text {bit }-r x}+E_{b i t-c o d}
$$

Where

$$
\begin{aligned}
& E_{\text {bit }-\mathrm{tx}}=\frac{P_{\mathrm{tx} x_{-}}\left(\frac{\text { header }+ \text { payload }+ \text { trailer }}{\text { rate }}\right)+P_{\mathrm{tx} \text { ini }} T_{\mathrm{tx} \text { ini }}}{\text { payload }} \\
& E_{\text {bit-out }}=\frac{P_{\text {tx_out }}\left(\frac{\text { header }+ \text { payload }+ \text { trailer }}{\text { rate }}\right)}{\text { payload }} d^{2} \\
& E_{\text {bit }-r x}=\frac{P_{r x_{-} e}\left(\frac{\text { header }+ \text { payload }+ \text { trailer }}{\text { rate }}\right)+P_{r x x_{-} \text {ini }} T_{r x_{-} \text {ini }}}{\text { payload }} \\
& E_{\text {bit -cod }}=\frac{E_{\text {coding }+} E_{\text {decoding }}}{\text { payload }}
\end{aligned}
$$

and

$E_{\text {bit }-t y}$ energy consumed per bit in electronic transmission

$E_{\text {bit-out }}$ : energy consumed per bit in output transmission

$E_{b i t-r x}$ : energy consumed per bit in electronic reception

$E_{b i t-c o d}$ : energy consumed per bit in coding/decoding processes

When our proposed algorithm decides to send a packet, the energy consumed by the sensor will be given 
by equation 6 :

$E_{\text {bit }-\mathrm{tx}}=k\left(E_{\text {bit-tx }}+E_{\text {bit }- \text { out }}+E_{\text {cod }}\right)+\mathrm{p}\left(E_{\text {bit }-r x}+E_{\text {dec }}\right)$

where $k$ is the size of the frame sent by the sensor, and $p$ is de size of the ACK sent back by the Gateway.

In order to save more energy, the system will use UDP protocol instead of TCP. This will reduce 96 bits the amount of bits transmitted, in addition to save the bits and time necessary to establish a TCP connection. Since the communication between sensors and gateway is using IEEE 802.15.4, UDP and IPv6, the minimum value of $k$ is 80 bytes, when a data frame sent by the sensor contains a one-byte parameter. In the other hand, the maximum frame size will by 84 bytes, when three one-byte parameters and 2-bytes parameter are sent. The size of the frame sent back by the gateway, for acknowledgment purpose, is 79 bytes, which consists of a frame with no data in the UDP payload. Under these conditions and considering a distance of 3 meters, the minimum of energy consumed per frame is 64.876 microJ and the maximum is 66.522 microJ.

\section{Interface}

In this section, the interface of our system is presented. An emoticon-based $\mathrm{HCI}$ in order to determine the wellness state of the user is proposed. The options, advanced settings menus and the habitability reports are also depicted. Also, the wellness state management algorithm is presented.

Different states are available. The selected states are 5, based on Likert's scale, from Feeling great as value 5 to Very bad as value 1 . The reason to use 5 different options is that it allows us to convert the qualitative data into quantitative data in order to analyse it as numbers ranging from 0 to 10 as it is presented. The proposed HCI is displayed in Fig. 5. We have focused on designing a minimalistic interface dynamic and easy to understand. The user can select easily the wellness state that better suits the current moment without consuming much of his/her time. Emoticons allow a better understanding of the state and engage the user in providing the wellness state as people are familiarized with employing them and understand their meaning. The name of the state is also written and different colors have been assigned to each state in order to avoid any doubts from the user, see Fig. 5.

The advanced settings allow the user to determine specific thresholds for the habitability parameters. The options available for the interface are presented in Fig. 6. The user can choose to enter the wellness state without being asked to do so, visualizing the daily report, the wellness report, the habitability report or selecting the advanced settings

Another feature of the advanced settings is the "At home hours" shown in Fig. 7. It allows the user to specify the hours he or she will be at home and can be notified about the request to enter the wellness state. Usually, in week days people have a regular schedule so the time can be specified for all the week days at once. The same happens with weekends. The blue box indicates the night hours the user is going to be at home and the yellow box is for the day-time. Other time ranges can be added selecting the plus button and introducing the time and the day of the week. This information is employed to shut the system down when the user is not at home, as there would be no change in the user as a consequence of the habitability parameters and the system would be consuming energy without obtaining information on the user.

The setting established in order to avoid disturbing the user when he or she is sleeping as well as for applying the conditions specified for the night is displayed in Fig. 8. It allows differentiating between weekdays and weekends. The display presented in Fig. 9 is an example of one of the parameters. As there are three ranges, from green to red depending on the disturbance placed on the user. Settings for day and night are different thus, two sets of maximum and minimum thresholds can be modified. The yellow box indicates that the threshold is for day-time and the blue one indicates that the threshold is for night-time. The system is configured with our proposed values but users are able to modify the settings at any time in order to better adjust the system to their needs. This information is then employed to generate some statistics about the progress of the user. 
The display for the habitability report sorted by parameter is presented from Fig. 10 to Fig.13. Fig. 10 presents the temperature report. It can be seen how the temperature changes through the day in each room of the house. For instance, in this case, higher temperatures are reached through the evening and colder ones at the early morning. The report for the humidity is shown in Fig. 11. It can be seen that the humidity for that day declined in the morning and then, increased again to start decreasing greatly again.

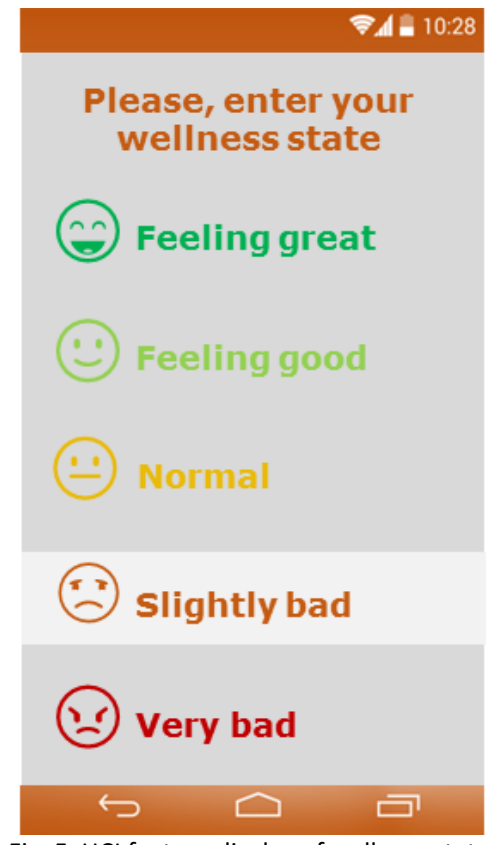

Fig. 5. $\mathrm{HCl}$ feature display of wellness state.

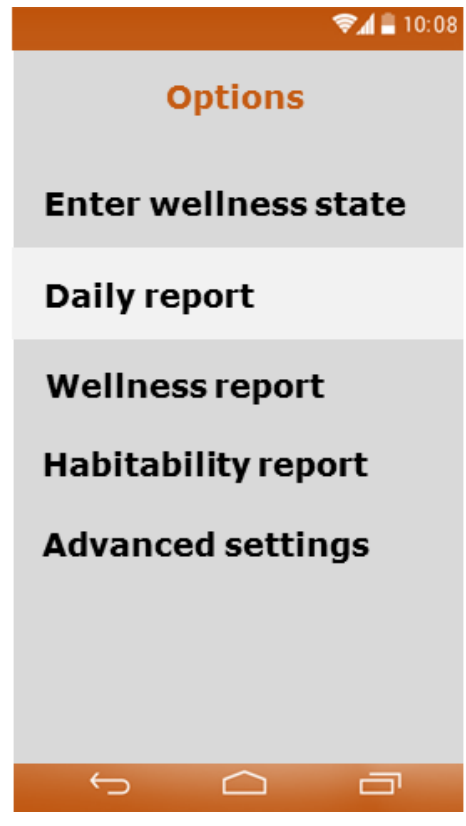

Fig. 6. $\mathrm{HCl}$ feature for options menu.

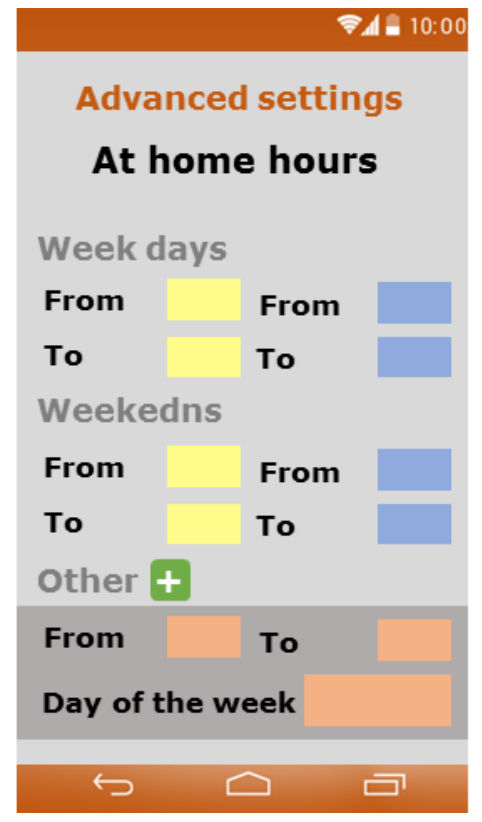

Fig. 7. $\mathrm{HCl}$ display for advanced settings.

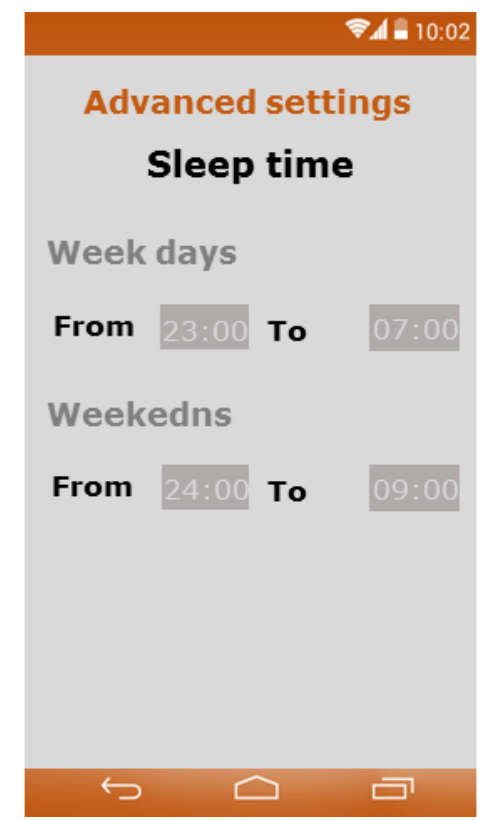

Fig. 8. $\mathrm{HCl}$ feature display for sleep time.

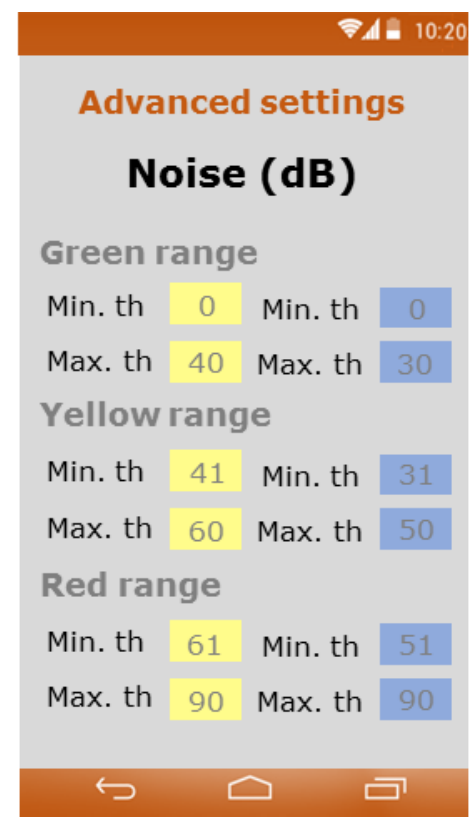

Fig. 9. $\mathrm{HCl}$ feature display for Noise. 


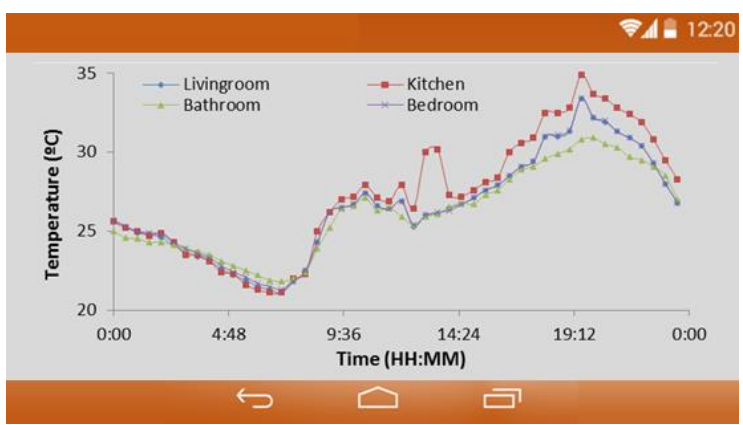

Fig. 10. Temperature habitability report of the $\mathrm{HCl}$.

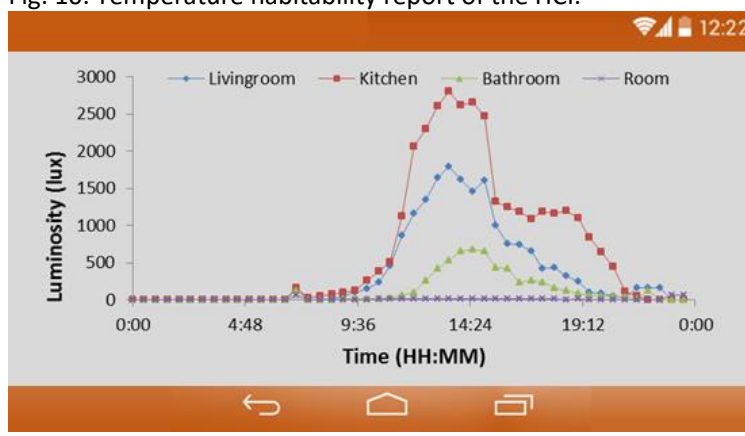

Fig. 12. Luminosity habitability report of the $\mathrm{HCl}$.

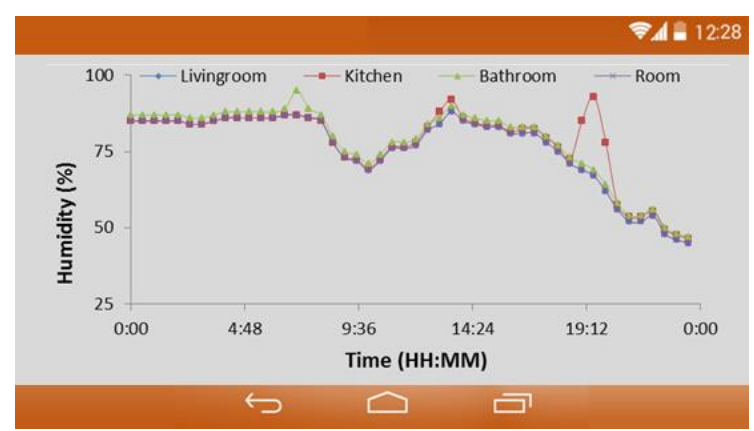

Fig. 11. Humidity habitability report of the $\mathrm{HCl}$.

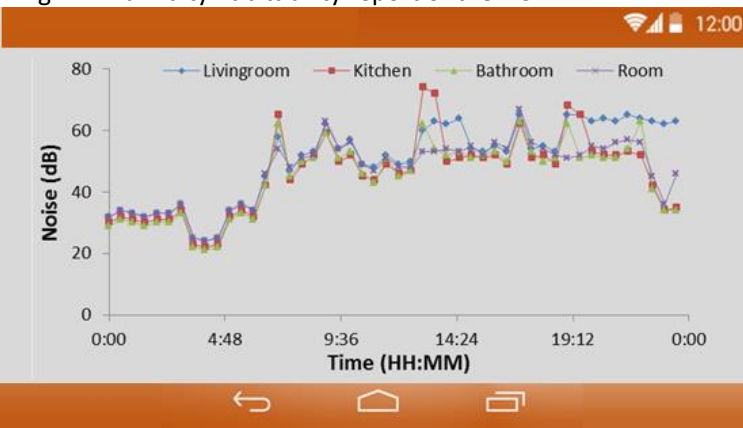

Fig. 13. Noise habitability report of the $\mathrm{HCl}$.

The luminosity report is displayed in Fig. 12. As it can be seen, there is a big increment during the day. The kitchen reaches higher levels of luminosity, partly because it is more exposed to daylight. That also influences the temperature, which is higher than the other rooms of the house. The noise report is presented in Fig. 13. It is noticeable that this parameter fluctuates more than the other ones. Also, noise at night is considerable lower than during the day. The changes in the noise are related to road traffic and the everyday life activity of the inhabitants of the dwelling. These graphs show the information for a user who spends 24 hours at home. For a normal day when the user goes to work and leaves the house, there would be less information.

The habitability report sorted by room, in this case the kitchen, is presented in Fig. 14 a) and b). The results are the same as the ones presented from Fig. 10 to Fig. 13 but, in this case, the figure displays the evolution of temperature, humidity, luminosity and noise for one room. This allows comparing all the parameters at the same time in order to have a quick overview of the conditions of a room. Although the parameters are not related, this graph allows to easily scan the condition of one room without going through different menus.

The algorithm for the wellness state management is presented in Fig 15. When starting the system, the user is asked about his or her wellness state in order to have an initial value. Then, if a change in the habitability parameters occurs, the user is requested to enter his or her wellness state. After that, the statistics are uploaded and the algorithm evaluates again if a new habitability value has been received. Even if a change has not occurred, the user can provide the wellness state if wanted. The statistics are then uploaded.
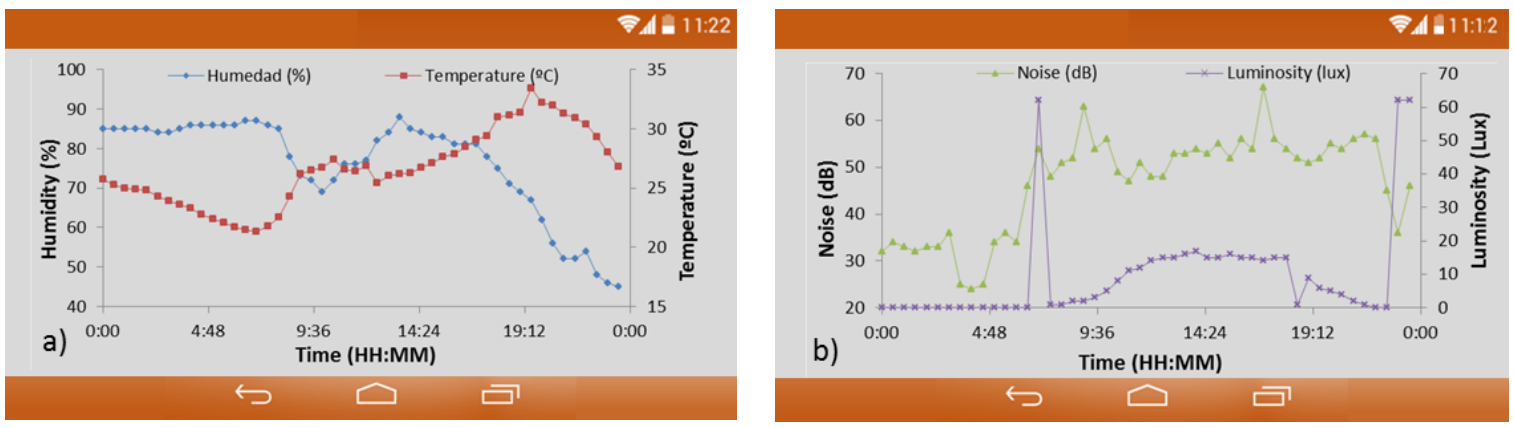

Fig. 14. Habitability report sorted by room. 


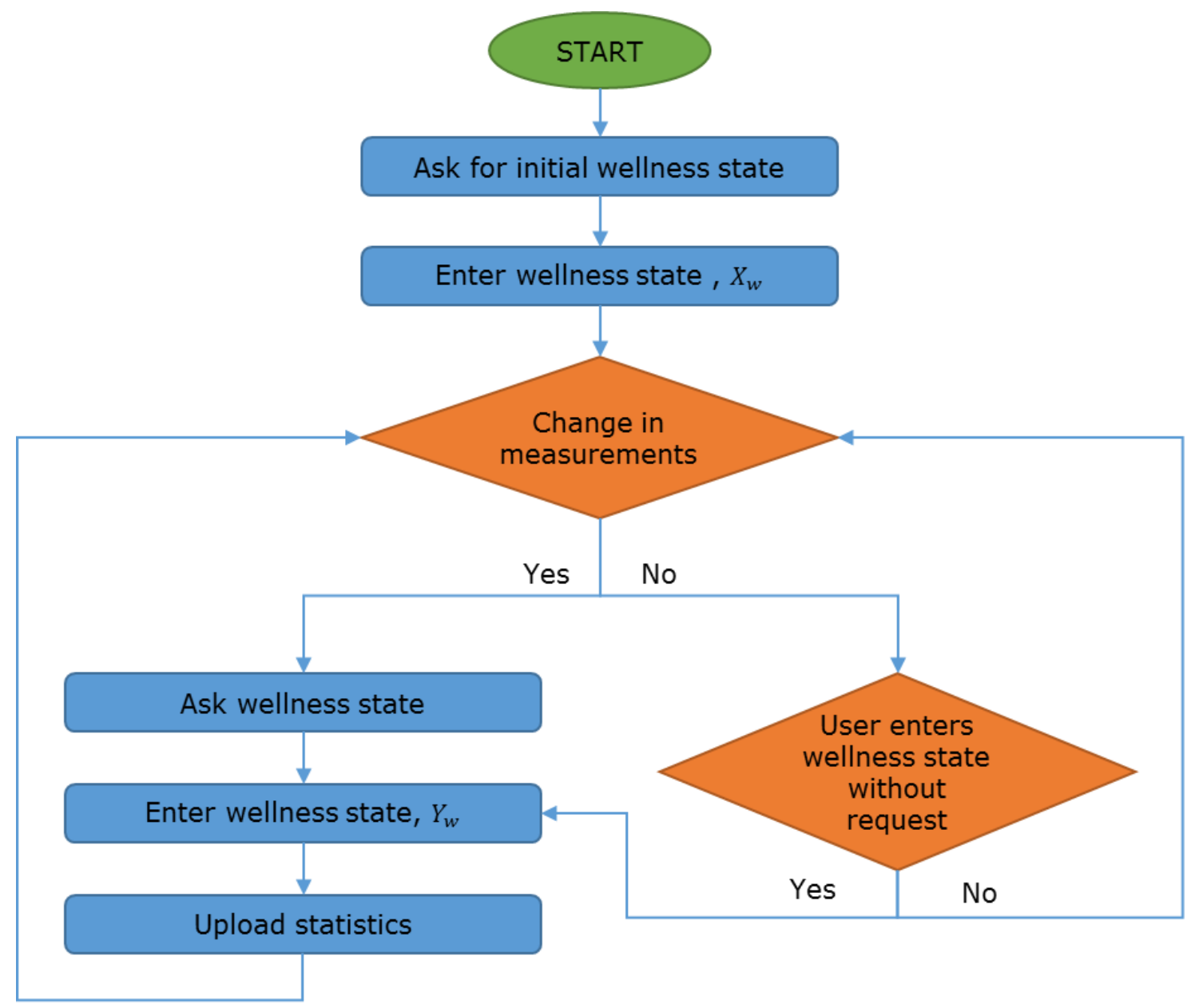

Fig. 15. Wellness state management algorithm.

\section{Results}

In this section, the results of employing our proposal in a real scenario are discussed. The sensors were placed in a house and the data was gathered during one day.

The tests were done in a small unipersonal studio with two rooms with windows overlooking the street, being one bedroom and the living-room. The other rooms, which are the kitchen and the bathroom, have windows overlooking the light well. The layout of the house can be seen in Fig. 16. The red spots represent the sensors, see Fig. 17, while the blue spot represents the gateway location. In each Ardunio node four different sensors are connected to sense the temperature, the sound, the humidity and the luminosity. The nodes are placed along the entire home; there is one sensor in each room as can be seen in Fig. 16. The nodes are wireless connected to the Gateway (see Fig. 16) using WiFi technology. The Gateway is placed in the Livingroom and it is almost equidistant to the nodes. The house is placed in a medium-concurrence road. During the measured period, no neighbors were living in the surrounding studios. The coordinates of the house are the following: $38^{\circ} 55^{\prime} 07.5^{\prime \prime} \mathrm{N} 0^{\circ} 06^{\prime} 52.8^{\prime \prime} \mathrm{W}$. The measures were toked during the $28^{\text {th }}$ of June. No air conditioner machines were used during the data gathering period in order to obtain as much real data as possible; however, the lights of different rooms were used. Only one person was living at the house during the measures and she followed the routine of her everyday life.

In order to test the performance of our proposed system and its operational algorithm the changes on the sensed parameters are shown. First, we need to study the changes of each variable during a short period of time in order to justify the different sensing period of each parameter. In Fig. 18 and Fig. 19 we can see the changes in the living room of each variable for 1 minute when nobody is at home. On the one hand, it is possible to see that humidity and temperature remain almost constant, with a standard deviation of 0.58 
and 0.24 respectively. On the other hand, luminosity and noise have a lot of variation. Luminosity had a lot of changes of \pm 1 lux from the central value of 12 lux, the standard deviation of the gathered data is 6.29. Finally, noise has huge variations due to the changes in road traffic. The maximum value is $51 \mathrm{~dB}$ and the minimum is $29 \mathrm{~dB}$. Based on those initial values, different sensing times for each variable are set. Luminosity and noise will need $0.1 \mathrm{~s}$ of monitoring each, as it is done in this test, in order to obtain as much information as possible. In contrast, the values for temperature and humidity will be monitored each minute thus $\mathrm{t}_{1}=1$.

To confirm that the proposed sensing period for humidity and temperature are enough, new data of temperature and humidity were gathered during 20 minutes. The data can be seen in Fig. 20 . The data was taken during the late afternoon just before it started to rain. The maximum temperature is $30.7^{\circ} \mathrm{C}$ and the minimum temperature is $25.71^{\circ} \mathrm{C}$. The minimum humidity is $46.2 \%$ and the maximum is $48.6 \%$.
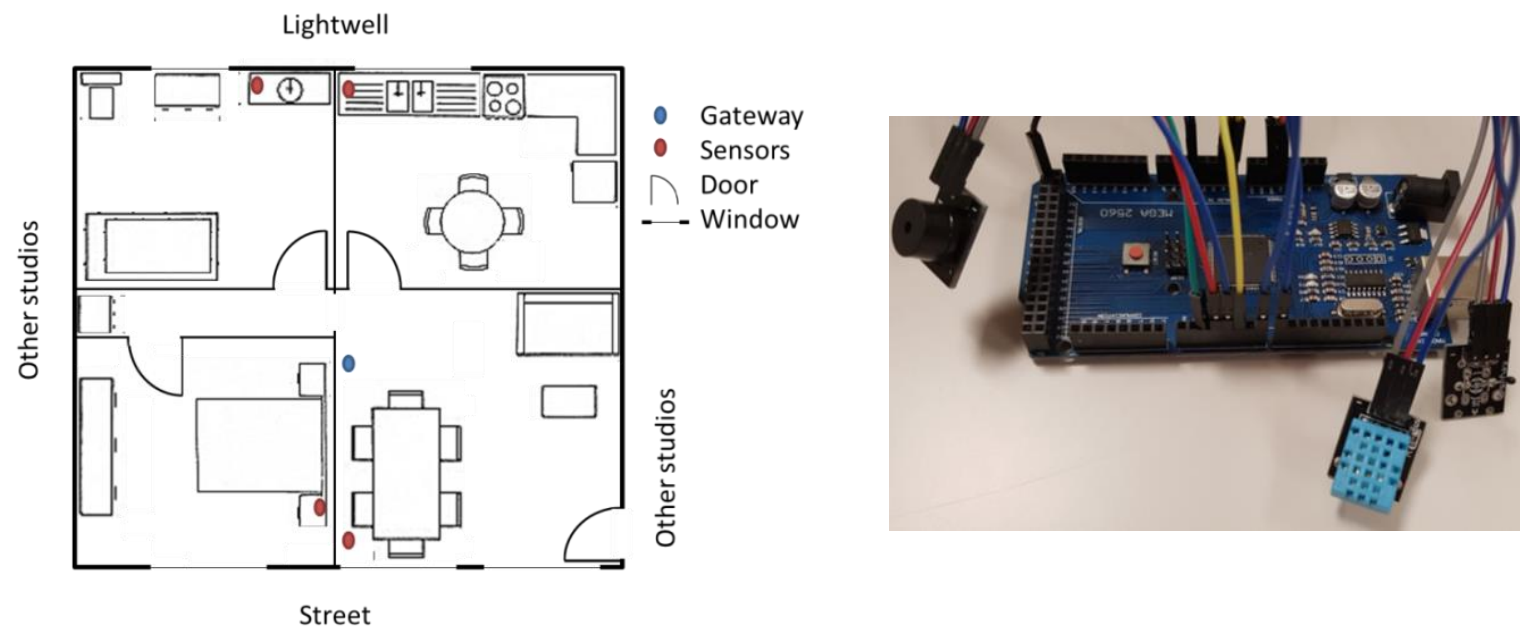

Fig. 16. Plan of the studied house

Fig. 17. Node and sensors used

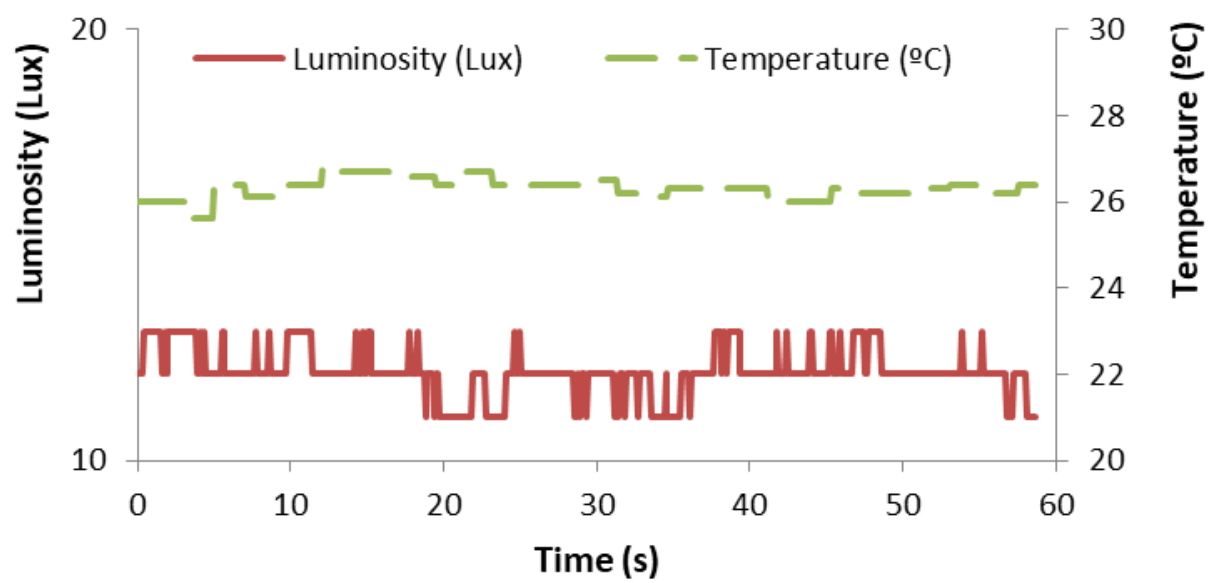

Fig. 18. Data gathered for luminosity and temperature in the preliminary test of all variables. 


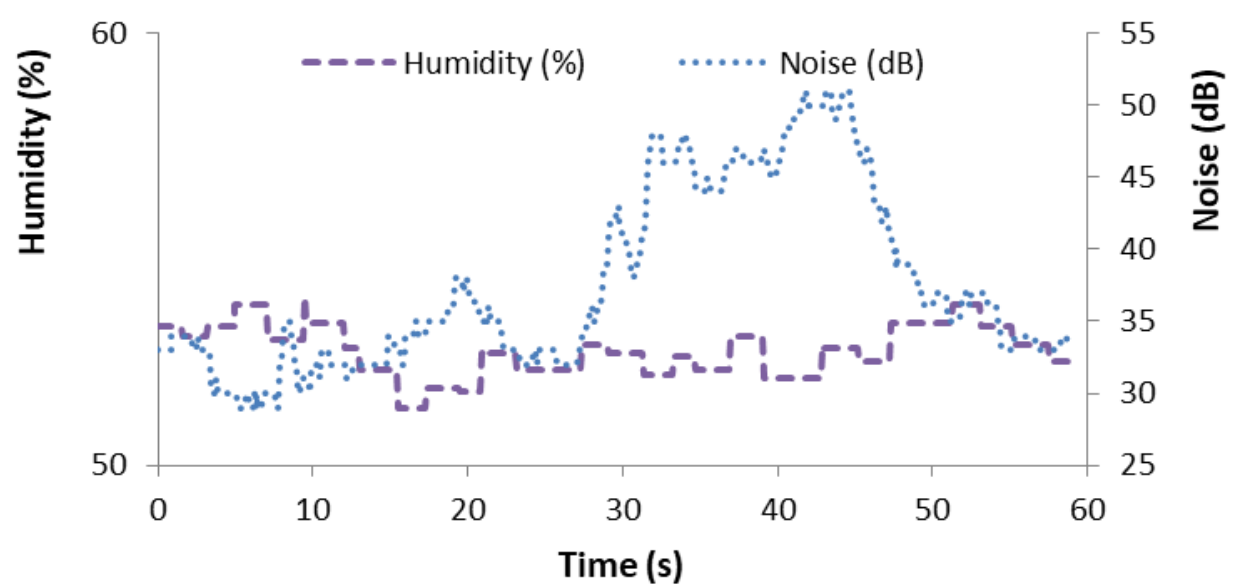

Fig. 19. Data gathered for humidity and noise in the preliminary test of all variables.

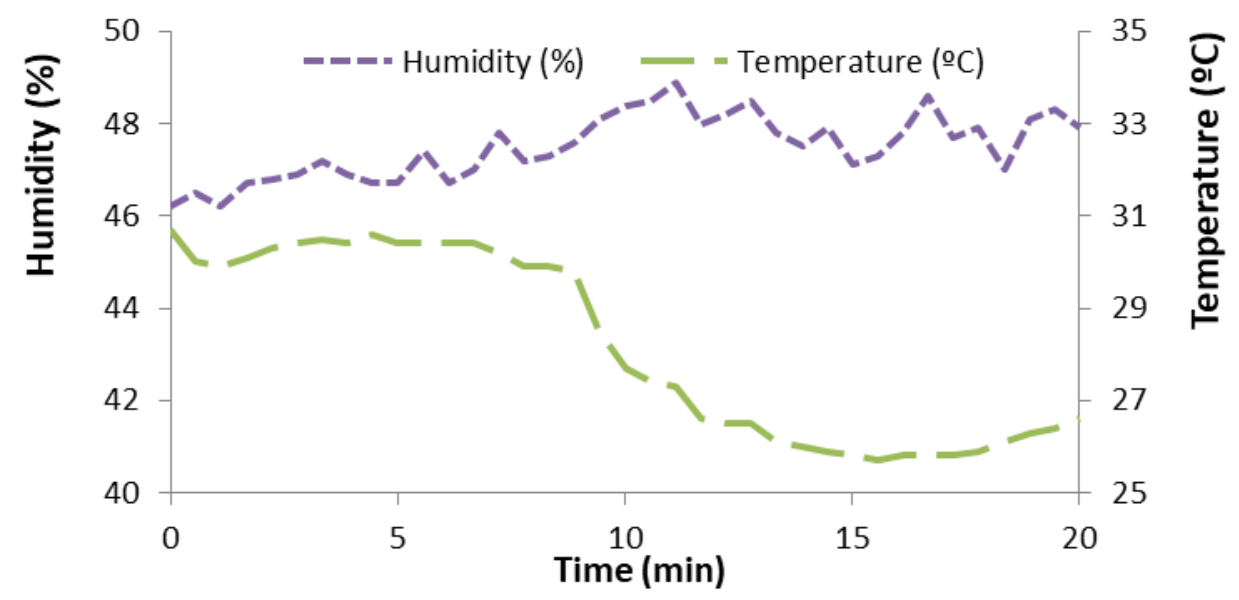

Fig. 20. Data gathered in the preliminary test of temperature and humidity

Now, the results of applying the protocol in different moments are shown. As the variable that changes the most is noise, the protocol is tested with noise data. Four different noise data sets with different changing rates are used. The data was gathered during $58.7 \mathrm{~s}$.

First, the data that would be registered by system with and without employing our algorithm is going to be determined. Four different options are modelled to send the data, the traditional forwarding with no algorithm (N.A.), or the other options which are: (i) $t_{2}=10$, (ii) $t_{2}=50$ and (iii) $t_{2}=100$. This corresponds to time intervals of 1s, 5s and 10s. Fig. 13, Fig. 14, Fig. 15 and Fig.16 present the available data in the system with the different sending options, grey with NA, red with $\mathrm{t}_{2}=10$, blue with $\mathrm{t}_{2}=50$ and green with $t_{2}=100$. In Fig. 21 the data gathered in the kitchen is shown. Because of the situation of the kitchen, few changes on noise occurred during the period of data gathering. Thus, the changes on noise are lower than the threshold of $5 \mathrm{~dB}$ and the data is sent only one time when the algorithm is used. For this reason, the available data in the system is the maximum value in $\mathrm{dB}$ during the first $1 \mathrm{~s}, 5 \mathrm{~s}$ or $10 \mathrm{~s}$, according to the selected $\mathrm{t}_{2}$

The data gathered by the sensor placed in the bathroom can be seen in Fig 22. In this case, the changes are greater than in the previous case and they overcome the threshold, so more data is sent. It is possible to see how the data available in the system follows the pattern of the data gathered without the algorithm. In this case different data are sent to the system in different moments when the algorithm is employed to update the available data. The result of applying the algorithm with the data gathered in the bedroom can be seen in Fig. 23. In this case, even greater changes than in the bathroom happened during the data gathering. The reason is the location of the bedroom. It is closer to the street than the kitchen and the bathroom. Again, it is possible to see how the data available in the system reproduces the changes of the real data. Finally, Fig. 24 presents the results with the data gathered in the living room. It is in this room where the highest number of changes occurred. Although some data may not be forwarded, the system is able to obtain all the information. That occurs because, when employing the algorithm, the collected data 
is highly similar to the real data.

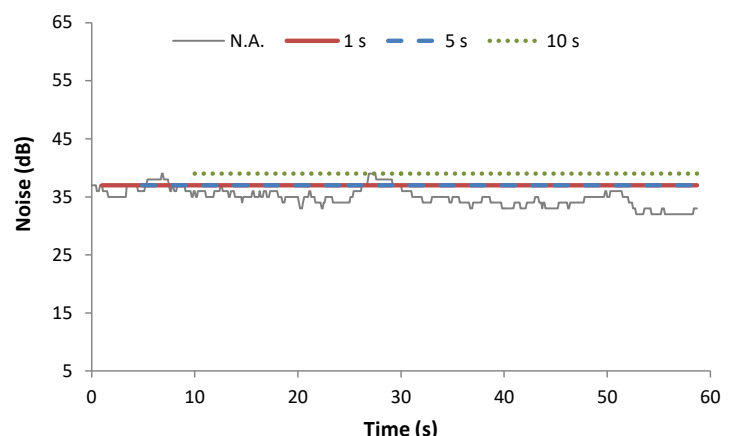

Fig. 21. Results of applying the algorithm with real data from kitchen.

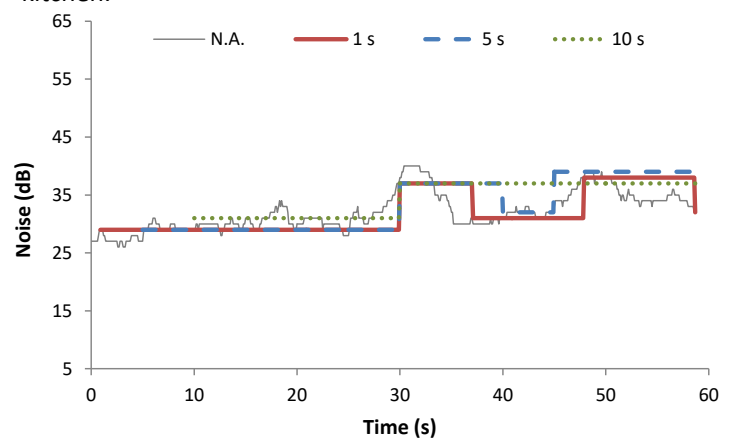

Fig. 23. Results of applying the algorithm with real data from bedroom.

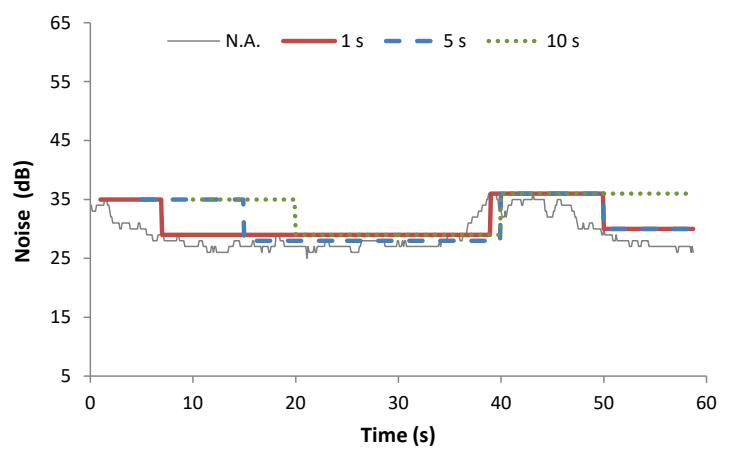

Fig. 22. Results of applying the algorithm with real data from bathroom.

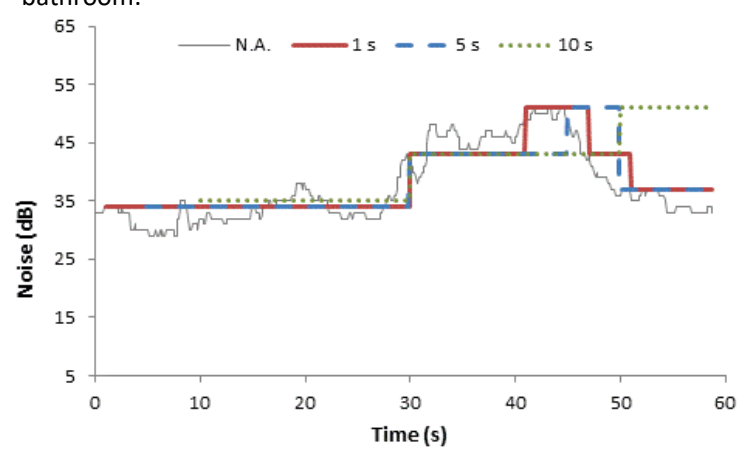

Fig. 24. Results of applying the algorithm with real data from living room.

The generated traffic of our proposed system, when applying the algorithm with different $t_{1}$ and $t_{2}$ and without applying the algorithm, can be seen in Fig. 25. For this data, we consider the sent packets of the previous cases, Fig. 21 to Fig. 24, that are corresponded to the four sensors placed at the house and the ACK from the gateway. In the worst case, when the four sensors gather data that changes in comparison to the previous data, our system generates the same traffic that the system without the algorithm, 636bytes/s. However, in most of the cases the traffic generated by our system with the algorithm is Obytes/s, while the proposal without the algorithm generates 636bytes of traffic each $0.1 \mathrm{~s}$. The total generated traffic during the entire test is $2.385 \mathrm{~kb}$ with $\mathrm{t} 2=10,2.067 \mathrm{~kb}$ with $\mathrm{t} 2=50$, and $1.431 \mathrm{~kb}$ with $\mathrm{t} 2$ $=100$. By the other side, without the algorithm the generated traffic is $373 \mathrm{~kb}$. The proposal results in a high reduction in the traffic while the available data reproduces accurately the data without algorithm.

Finally, the consumed energy of the proposed scenario is shown in Fig. 26. The consumed energy is represented as the accumulated consumed $\mathrm{mJ}$ in time. The energy consumption of all the nodes is considered, but not the energy consumed by the gateway. As in the generated traffic, our system with the algorithm supposes a huge energy saving. Not only because the reduction in the sending time, $0.1 \mathrm{~s}$ without algorithm or $1 \mathrm{~s}, 5 \mathrm{~s}$ and $10 \mathrm{~s}$ with the algorithm, also because data is not forwarded each possible sending time. In the one hand, in the case that N. A. is used, each $0.1 \mathrm{~s}$ a total of $0.259504 \mathrm{~mJ}$ are consumed in the system. The total consumed energy with N. A. during the $58.7 \mathrm{~s}$ is $152.59 \mathrm{~mJ}$. On the other hand, when the algorithm is used the total energy consumed to send the data is reduced to less than $1 \mathrm{~mJ}$ in all the cases. The consumed energy with $t_{2}=10$ is $0.973 \mathrm{~mJ}$, with $t_{2}=50$ is $0.843 \mathrm{~mJ}$, and with $t_{2}=$ 100 is $0.584 \mathrm{~mJ}$. That implies a $99.36 \%, 99.45 \%$, and $99.62 \%$ of energy saving for $\mathrm{t} 2=10$, $\mathrm{t} 2=50$ and $\mathrm{t} 2$ $=100$ respectively. 


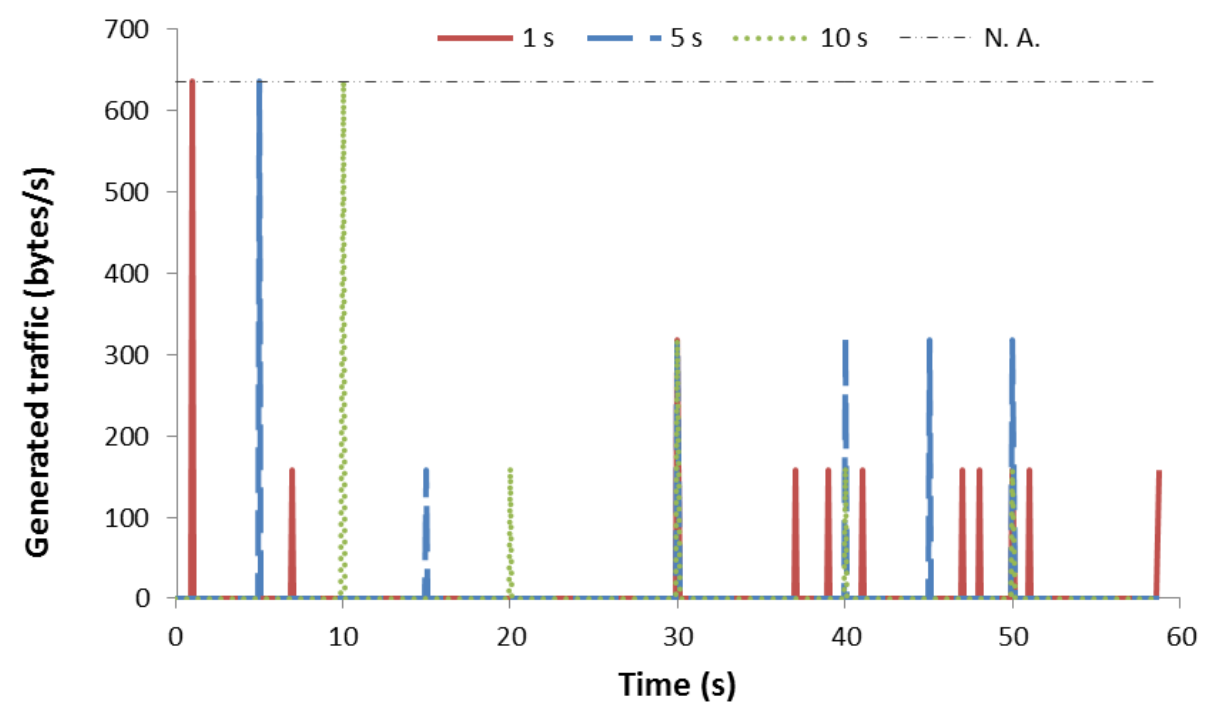

Fig. 25. Results of apply the algorithm with real data from living room.

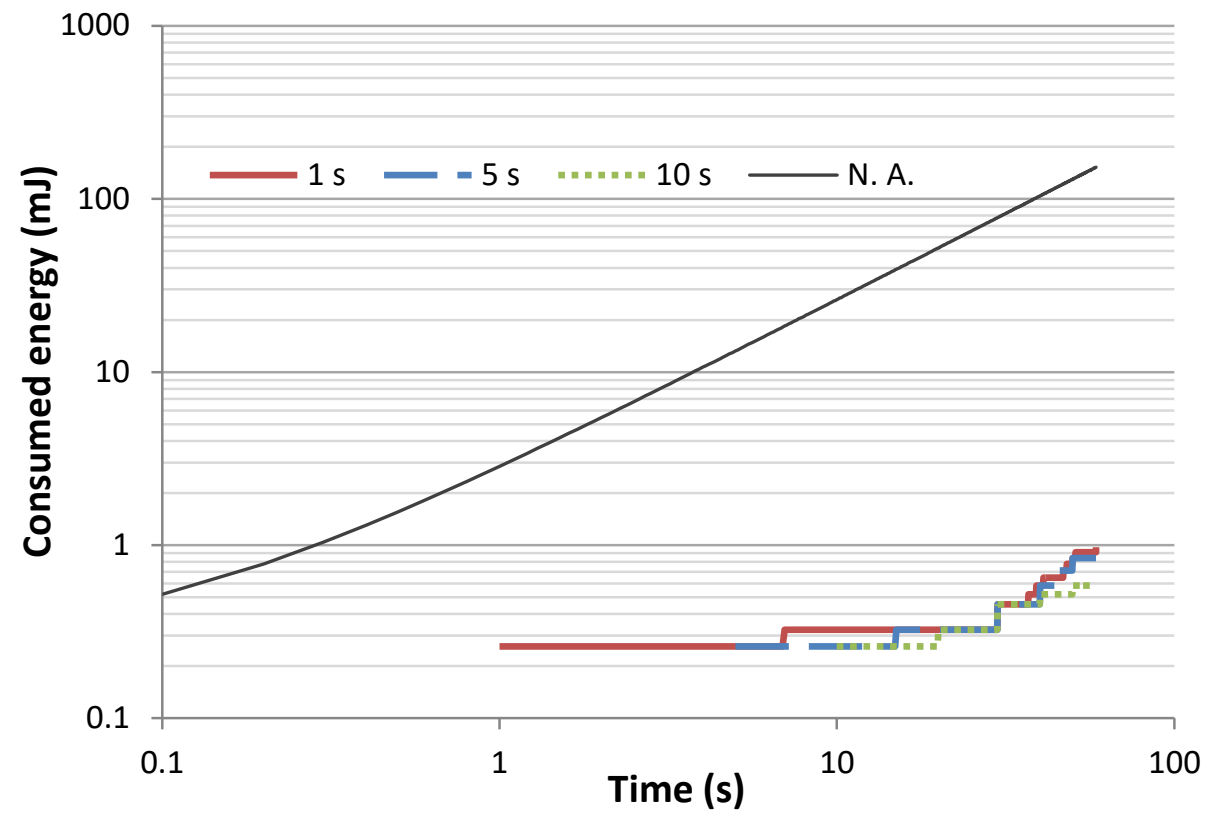

Fig. 26. Results of apply the algorithm with real data from the bedroom.

\section{CONCLUSION AND FUTURE WORK}

In this paper, we have presented our system for monitoring the wellness state of the inhabitants of a dwelling based on the habitability parameters measured at the house. We also propose an emoticon-based $\mathrm{HCI}$ in order to gather the data on the wellness state of the user. A forwarding decision-making algorithm was designed to measure and determine when to send the gathered information to the database. Tests in a real domestic environment were performed in order to determine the best settings for the forwarding decision-making. Also, the energy consumption was measured obtaining $0.973 \mathrm{~mJ}, 0.843 \mathrm{~mJ}$ and $0.584 \mathrm{~mJ}$ for $t_{2}=10, t_{2}=50$ and $t_{2}=100$ respectively. The energy consumption without utilizing the algorithm was $152.59 \mathrm{~mJ}$, resulting in an improvement in energy consumption of $99.36 \%$ for $\mathrm{t} 2=10,99.45 \%$ for $\mathrm{t} 2=50$, and $99.62 \%$ for $\mathrm{t} 2=100$. Considering the results, we have decided to employ $t_{2}=50$ because with higher values too much valuable information is lost. Also, both $t_{2}=10$ and $t_{2}=50$ lead to similar results so we have determined that $t_{2}=50$ is the best value.

As future work, we are planning to employ sensors for ambient assisted living monitoring [45] to measure subjective wellness state parameters. That way, we will statistically study the correlation between habitability and wellness conditions in order to provide a better assessment of the quality of life of the 
user. Also, we are planning to adapt our system to different conditions of the user, for example, people with chronic illnesses, in order to develop several alarms to detect if the state of the user is getting worse. We also want to provide artificial intelligence to our system in order to recommend the room with the best conditions for the user, as we have previously done in [26].

\section{References}

[1] S Sendra, L Parra, J Lloret and J Tomás, "Smart System for Children's Chronic Illness Monitoring", Information Fusion, Vol. 40, pp. 76-86, Mar. 2017.

[2] J Lloret, L Parra, M Taha and J Tomás, “An architecture and protocol for smart continuous eHealth monitoring using 5G", Computer Networks, Elsevier, May 2017, In press. DOI: http://doi.org/10.1016/j.comnet.2017.05.018

[3] B. Hettler, "The six dimensions of wellness", National Wellness Institute, 1976. Available: http://c.ymcdn.com/sites/www.nationalwellness.org/resource/resmgr/docs/sixdimensionsfactsheet.pdf

[4] H. L. Dunn, "What High-Level Wellness Means”, Canadian Journal of Public Health, Vol. 50, no. 11, pp. 447-457, Nov. 1959.

[5] D.J. Herbes and C. H. Mulder, "Housing and subjective well-being of older adults in Europe", Journal of Housing and the Built Environment, Springer, pp. 1-26, Oct. 2016. DOI: 10.1007/s10901016-9526-1

[6] OECD, “How's Life? Measuring Well-being”, 2015. Available at: http://www.oecdilibrary.org/economics/how-s-life_23089679;jsessionid=55pjippucpjrq.X-oecd-live-02

[7] G.C Donaldson, T. Seemungal, D.J. Jeffries and J. A. Wedzicha, "Effect of temperature on lung function and symptoms in chronic obstructive pulmonary disease", European Respiratory Journal, ERS, Vol. 13, No. 4, pp. 844-849, Apr. 1999.

[8] J. Schwartz, J. Samet and J. Patz, "Hospital Admissions for Heart Disease: The Effects of Temperature and Humidity”, Epidemiology, Wolters Kluwer, Vol. 15, No. 6, pp. 755-761, Nov. 2004.

[9] National Institute of Statistics of Spain, "Defunciones según causa de muerte en 2003", Nov. 2005. Available at: http://www.ine.es/prensa/np393.pdf

[10] A. Grimes, T. Denne, P. Howden-Dhapman, R. Arnold, L. Telfar-Barnard, N. Preval and C. Young, "Cost Benefit Analysis of the Warm Up New Zealand: Heat Smart Programme", Wellington: Universit of Wellington, 2012. Available at: http://sustainablecities.org.nz/wpcontent/uploads/NZIF_CBA_report2.pdf

[11] B. Martínez-Pérez, I. de la Torre-Díez, S. Candelas-Plasencia and M. López-Coronado, "Developement and Evaluation of Tools for Measuring the Quality of Experience (QoE) in mHealth Applications", Journal of Medical Systems, Springer, Vol. 37, No. 5, pp.9976, Sep. 2013.

[12] J. B. Walther and K. P. D'addario, "The Impacts of Emotions on Message Interpretation in Computer-Mediated Communication", Social Science Computer Review, SAGE, Vol. 19, No. 3, pp. 324-347, Aug. 2001.

[13] H. Ghayvat, J. Liu, S. C. Mukhopadhay and X. Gui, "Wellness Sensor Networks; A Proposal and Implementation for Smart Home for Assisted Living”, IEEE Sensors Journal, IEEE, Vol. 15, No. 12, pp. 7341-7348, Dec. 2015.

[14] A. R. M. Forkan and W. Hu, "A context-aware, predictive and protective approach for wellness monitoring of cardiac patients", on Computing in Cardiology Conference, Vancouver, Canada, 11-14 September, 2016, pp. 369-372.

[15] C. E. R. Booc, C. M. D. San Diego, M. L. Tee and J. D. L. Caro, "A mobile application for campusbased psychosocial wellness program", 7th International Conference on Information, Systems \& Applications, Chalkidiki, Greece, 13-15 July, 2016, pp. 1-4.

[16] W. A. Khan, M. Idris, T. Ali, R. Ali, S. Hussain, M. Hussain, M. B. Amin, A. M. Khattak, Y. Weiwei, M. Afzal, S. Lee and B. H. Kang, "Correlating health and wellness analytics for personalized decision making”, Boston, USA, 14-17 October, 2015, pp. 256-261.

[17] C. Lim, Z. M. Kim and H. Choi, "Context-based healthy lifestyle recommendation for enhancing user's wellness", IEEE International Conference on Big Data and Smart Computing, Jeju, South Korea, 13-16 February, 2017, pp. 418-421. 
[18] B. Tulu, D. Strong, L. Wang, Q. He, E. Agu, P. Pedersen and S. Djamasbi, "Design Implications of User Experience Studies: The Case of a Diabetes Wellness App", 49th Hawaii International Conference on System Sciences, Koloa, USA, 5-8 January, 2016, pp. 3473-3482.

[19] D. Kaur and G. S. Siddaraju, "Experimental study of cardiac functionality for the wellness of individual by developing an android application", International conference on Computation System and Information Technology for Sustainable Solutions, Bangalore, India, 6-8 October, 2016, pp. 174-183.

[20] A. Arshad, S. Khan, A. H. M. Z. Alam, R. Tasnim and R. I. Boby, "Health and Wellness Monitoring of Elderly People Using Intelligent Sensing Technique", International Conference on Computer and Communications Engineering, 26-27 July, 2016, Kuala Lumpur, Malaysia, pp. 231-235.

[21] C. J. Martin, S. D. Platt and S. M. Hunt, "Housing conditions and ill health", British Medical Journal, Clin Res Ed, Vol. 294, No. 6580, pp. 1125-1127, May 1987.

[22] G. W. Evans, N. M. Wells and A. Moch, "Housing and Mental Health: A Review of the Evidence and a Methodological and Conceptual Critique", Journal of Social Issues, Vol. 59, No. 3, pp. 475-500, 2003.

[23] M. Shaw, "Housing and public health", Annual Review of Public Health, Vol. 25, pp. 397-418, Apr. 2004.

[24] H. Thomson and S. Thomas, "Developing empirically supported theories of change for housing investment and health", Social Science \& Medicine, Springer, Vol. 124, pp. 205-214, Jan. 2015.

[25] C. J. Gustafson, S. R. Feldman, S. A. Quandt, S. Isom, H. Chem, C. R. Spears and T. A. Arcury, "The association of skin conditions with housing conditions among North Carolina Latino migrant farm workers", International Journal of Dermatology, Vol. 53, No. 9, pp. 1091-1097, Sep. 2014.

[26] R. Laquesta, L. Garcia, I. Garcia-Magarino and J. Lloret, "System to Recommend the Best Place to Life Based on Wellness State of the User Employing the Heart Rate Variability”, IEEE Access, Vol. 5, pp. 10594-10604, May 2017.

[27] F. Isiaka, K. Mwitondi and A. Ibrahim, "Automatic Prediction and Detection of Affect State Based on Invariant Human Computer Interaction and Human Physiological Response”, Seventh International Conference on Computational Intelligence, Modelling and Simulation, Kuantan, Malaysia, 27-29 July, 2015, pp. 19-25.

[28] S. Han, R. Liu, C. Zhu, Y. G. Soo, H. Yu, T. Liu and F. Duan, "Development of a human computer interaction system based on multi-modal gaze tracking methods", IEEE International Conference on Robotics and Biomimetics, Qingdao, China, 3-7 December, 2016, pp. 1894-1899.

[29] B. Chen, S. Huang and W. Tsai, "Eliminating Driving Distractions: Human-Computer Interaction with Built-In Applications”, IEEE Vehicular Technology Magazine, IEEE, Vol. 12, No. 1, pp. 20-29, Mar. 2017.

[30] S. Kamal, F. Sayeed and M. Rafeeq, "Facial emotion recognition for Human-Computer Interactions using hybrid feature extraction technique", International Conference on Data Mining and Advanced Computing, Ernakulam, India, 16-18 March, 2016, pp. 180-184.

[31] R. Agrawal and N. Gupta, "Real Time Hand Gesture Recognition for Human Computer Interaction", IEEE 6th International Conference on Advanced Computing, Bhimavaram, India, 27-28 February, 2016, pp. 470-475.

[32] C. S. Sánchez, A. Mavrogianni and F. J. N. González, "On the minimal thermal habitability conditions in low income dwellings in Spain for a new definition of fuel poverty", Building and Environment, Elsevier, Vol. 114, pp. 344-356, Mar. 2017.

[33] Ministry of Health, Social Services and Equality of Spain, "Plan Nacional de Actuaciones Preventivas de los Efectos del Exceso de Temperaturas Sobre la Salud”, 2015. Available at: http://www.msssi.gob.es/ciudadanos/saludAmbLaboral/planAltasTemp/2015/docs/Plan_Nacional_de_E xceso_de_Temperaturas_2015.pdf

[34] C. G. Bornehag, G. Blomquist, F. Gyntelberg, B. Järvholm, P. Malmberg, L. Nordvall, A. Nielsen, G. Pershagen and J. Sundell, "Dampness in buildings and health", Indoor air, Vol. 11, No. 2, pp. 72-86, Jun. 2001.

[35] M. H. Garret, P. R. Rayment, M. A. Hooper, M. J. Abramson and B. M. Hooper, "Indoor airborne fungal spores, house dampness and associations with environmental factors and respiratory health in children”, Clinical and Experimental Allergy, Blackwell Science Ltd., Vol. 28, pp. 459-467, Nov. 1997. 
[36] M. B. C. Ariës nad L. Zonneveldt, "Architectural Aspects of Healthy Lighting”, 21th Conference on Passive and Low Energy Architecture, The Netherlands, 19-22 September, 2004, pp. 1-5.

[37] M. Boubekri, I. N. Cheung, K. J. Reid, C. Wang and P. C. Zee, "Impact of windows and daylight exposure on overall health and sleep quality of office workers: A case-control pilot study", Journal of Clinical Sleep Medicine, Vol. 10, No. 6, pp. 603-611, Jun. 2014.

[38] F. Beute and Y. A. W. de Kort, "Salutogenic Effects of the Environments: Review of Health Protective Effects of Nature and Daylight", Applied psychology: Health and Well-being, Vol. 6, No. 1, pp. 67-95, 2014.

[39] P. Boyce, C. Hunter and O. Howlett, "The benefits of daylight through Windows", Troy, New York, Rensselaer Polytechnic Institute, Sep. 2003.

[40] W. J. G. Hoogendijk, P. Lips, M. G. Dik, D. J. H. Deeg, A. T. F. Beekman and B. W. J. H. Penninx, "Depression is associated with decreased 25-Hydroxyvitamin D and increased Parathyroid hormone levels in older adults", Archives of general psychiatry, Vol. 65, No. 5, pp. 508-512, 2008.

[41] H. Ising and B. Kruppa, "Health effects caused by noise: Evidence in the literature from the past 25 years", Noise \& Health, Vol. 6, No. 22, pp. 5-13, 2004.

[42] S. Sandra, J.Lloret, M. Garcia, J.F. Toledo, "Power saving and energy optimization techniques for Wireless Sensor Networks", Journal of Communications, Vol. 6, No. 6, 2011.

[43] W. R. Heinzelman, A. Chandrakasan, A., H. Balakrishnan, "Energy-Efficient Communication Protocol for Wireless Microsensor Networks", In Proceedings of the IEEE 33rd annual Hawaii international conference on System Sciences, Maui, Hawaii, January 4-7, 2000,

[44] J.P. Kaps, B. Sunar, "Energy Comparison of AES and SHA-1 for Ubiquitous Computing", In Proceedings of the EUC 2006 Workshops: NCUS, SecUbiq, USN, TRUST, ESO, and MSA, Seoul, Korea, August 1-4, 2006 .

[45] L Parra, S Sendra, JM Jiménez and J Lloret, "Multimedia sensors embedded in smartphones for ambient assisted living and e-health", Multimedia Tools and Applications, Vol. 75, No. 21, pp. 1327113297, 2016. 